\title{
The expression profile of Fc Receptor-like Y in B lymphocytes with hepatitis B virus induced diseases
}

\author{
Ke Wang ${ }^{1}$, Run-Lin Yang ${ }^{1}$, Hao $\mathrm{Pei}^{2}$, Hang-Yuan $\mathrm{Wu}^{2}$, Jue Zhang ${ }^{1}$, Biao Huang ${ }^{1}$ \\ ${ }^{1}$ Key Laboratory of Nuclear Medicine, Ministry of Health, Jiangsu Key Laboratory of Molecular \\ Nuclear Medicine, Jiangsu Institute of Nuclear Medicine, Wuxi 214063, Jiangsu Province, China \\ ${ }^{2}$ Wuxi Hospital of Infectious Disease, Wuxi 214005, Jiangsu Province, China
}

\begin{abstract}
The Fc Receptor-like Y (FcRY) molecule is preferentially expressed by B lymphocytes and has recently been considered as a potential therapeutic target for B cell malignancies. In this study, we investigated the correlation between FcRY expression profile, B lymphocytes population and different HBV infection disease status. The FcRY expression level on B lymphocytes and the number of B lymphocytes population from peripheral blood in 27 healthy controls (HC) and 65 patients with HBV-induced diseases, including chronic hepatitis B (CHB), liver cirrhosis (LC) and hepatocellular carcinoma (HCC), were analyzed using quantitative real-time PCR and flow cytometry. The results showed the level of FcRY expression and frequency of germinal center (GC) B lymphocytes from peripheral blood were significantly correlated with the HBV-related disease status, which was highest in HCC and LC patients, lowest in healthy donors, and in the middle in patients with CHB. Our study indicates that there is a significant correlation between FcRY expression profile, B lymphocytes population and HBV-induced diseases. However, the roles of FcRY and B lymphocytes in HBV-induced diseases are unclear and need further investigation. (Folia Histochemica et Cytobiologica 2011; Vol. 49, No. 3, pp. 405-409)
\end{abstract}

Key words: Fc Receptor-like Y, B lymphocytes, germinal center B lymphocytes, hepatitis B virus, gene expression

\section{Introduction}

Hepatitis B virus (HBV) infection is highly prevalent worldwide, especially in the Asia-Pacific region, and is a major cause of morbidity and death. It is estimated that two billion people globally have been infected with HBV and tens of millions of new cases occur annually. Of those infected, $15 \%$ to $40 \%$ could develop into liver cirrhosis (LC) or hepatocellular carcinoma (HCC) [1-3]. Due to its significant economic and public health impact, the control and prevention of hepatitis B continues to be a major concern.

The cellular and molecular mechanisms of HBV infection have been investigated and it is now widely

Correspondence address: B. Huang,

Jiangsu Institute of Nuclear Medicine,

Wuxi 214063, Jiangsu Province, China;

tel.: (+ 86) 138617320 32, fax: (+ 86510$) 855207$ 70;

e-mail: cluseee@gmail.com accepted that the adaptive immune responses, particularly the cellular immune response, play key roles in the development of immunity to HBV.

CD4 T cells, classically referred to as helper T cells, are robust producers of cytokines, while CD8 T cells go on to clear HBV-infected hepatocytes through cytolytic and non-cytolytic mechanisms, reducing the levels of circulating virus $[4,5]$. Despite the cellular immune response being a major contributor to HBV clearance, humoral responses by B lymphocytes are also important in controlling HBV [6]. In addition to the production of antibodies, little is known about the role of B lymphocytes such as differentiation, maturation and function during the natural course of $\mathrm{HBV}$ infection.

Fc receptor-like (FCRL) molecules are a family of Fc receptor homologs comprising eight structurally related members [7-10]. Seven of the eight FCRL family members are preferentially expressed by B cells at different stages in their development, the excep- 
Table 1. Clinical characteristics of the subjects enrolled in the study

\begin{tabular}{|l|c|c|c|c|}
\hline Parameters & HC $(\mathbf{n}=\mathbf{2 7})$ & CHB $(\mathbf{n}=\mathbf{2 5})$ & LC $(\mathbf{n}=\mathbf{2 0})$ & HCC $(\mathbf{n}=\mathbf{2 0})$ \\
\hline Age (years) & $35.9 \pm 7.2$ & $37.2 \pm 10.3$ & $44.5 \pm 10.1$ & $40.2 \pm 9.2$ \\
\hline Sex $(\mathrm{M} / \mathrm{F})$ & $10 / 6$ & $14 / 11$ & $7 / 3$ & $6 / 4$ \\
\hline Serum bilirubin $[\mathrm{mg} / \mathrm{mL}]$ & $0.6 \pm 0.4$ & $3.5 \pm 10.7$ & $22.4 \pm 10.1$ & $30.1 \pm 18.6$ \\
\hline Albumin [g/dL] & $4.5 \pm 1.9$ & $4.2 \pm 3.0$ & $6.3 \pm 3.9$ & $5.5 \pm 2.1$ \\
\hline AST [IU/L] & $19.3 \pm 3.2$ & $96.6 \pm 45.3$ & $116.0 \pm 55.7$ & $104.2 \pm 62.7$ \\
\hline ALT [IU/L] & $15.4 \pm 2.8$ & $99.8 \pm 28.2$ & $98.8 \pm 43.3$ & $116.4 \pm 50.3$ \\
\hline
\end{tabular}

HC — healthy controls; CHB — chronic hepatitis B; LC — liver cirrhosis; HCC — hepatocellular carcinoma; AST — aspartate aminotransferase; ALT - alanine transaminase

tion being FCRL6 [11-17]. FcRY, also known as FREB2 and FCRLB, is one of the FCRL family members and found to be an intracellular protein consisting of three immunoglobulin-like domains and a carboxy terminal mucin-like domain. The analysis of FcRY expression within human tonsils indicates that this protein is mainly expressed in subset of germinal center (GC) B cells, which suggests that it may play an important role in regulating cellular differentiation and modulating the initiation and termination of B-cell responses [18-20].

Recent studies indicate that the activated level of $B$ cells in human peripheral blood lymphocytes with HBV infection is higher than that of normal controls [21]. Based on that finding, we hypothesize that HBV infection might alter the proportion of B cells subpopulation and thus influence the expression level of FcRY. In this report, we investigated the correlation within the expression profile of FcRY, B lymphocytes population and $\mathrm{HBV}$-induced diseases, especially chronic infection, LC and HCC, with peripheral blood. We believe that this report may provide valuable insights into $\mathrm{HBV}$-induced disease pathology.

\section{Material and methods}

Samples. This study was conducted with the approval of the local Ethics Committee of Wuxi Hospital of Infectious Disease and Jiangsu Institute of Nuclear Medicine. All donors gave written informed consent before blood was drawn. Ninety-two subjects, including 27 healthy controls and 65 patients infected with HBV, were recruited from Wuxi Hospital of Infectious Disease between 2008 and 2009 in the present study. The patients were divided into four groups according to the progression of HBV-induced diseases. The first group comprised 27 healthy controls with no previous evidence of any liver diseases and all serological HBV-viral markers tested negative. The second group was $25 \mathrm{CHB}$ patients, who were hepatitis B surface antigen (HBsAg)-positive for more than six months and had histological evidence of chronic hepatitis. The third group included 20
HBV-related LC patients, and the fourth group was $20 \mathrm{HBV}$ -related HCC patients clinically and histologically diagnosed. Clinicopathological data were retrospectively reviewed, which included age, sex, serum bilirubin, albumin, AST and ALT (Table 1).

Cell preparation. Heparinized peripheral blood samples $(10 \mathrm{ml})$ collected from the donors were diluted in PBS and layered on Isopaque-Ficoll separation medium and centrifuged for $30 \mathrm{~min}$ at $400 \times \mathrm{g}$. Peripheral blood mononuclear cells (PBMC) accumulating at the interface were separated and resuspended at RPMI 1640. CD19 B cells were isolated from PBMC using direct MACS kit (StemCell, Vancouver, BC, Canada) according to the manufacturer's instruction. The purity of cells was $>95 \%$ as determined by flow cytometry.

Real-time PCR. Total RNA was extracted from sorted cells using Trizol according to the manufacturer's recommendations and first-strand cDNA synthesis using PrimeScript $\mathrm{t}^{\mathrm{TM}}$ RT reagents Kit (Takara, Otsu, Shiga, Japan). Real-time polymerase chain reaction (RT-PCR) was performed with the cDNA using SYBR green PCR Master Mix (Takara, Otsu, Shiga, Japan). Each amplification reaction underwent denaturation at $95^{\circ} \mathrm{C}$ for $30 \mathrm{~s}$, amplification for 40 cycles at $95^{\circ} \mathrm{C}$ for $5 \mathrm{~s}$, annealing and extension at $60^{\circ} \mathrm{C}$ for $20 \mathrm{~s}$ using LightCycler sequence detection system. Human FcRY gene-specific primers used for real-time PCR amplification were 5'GGG AGC ACC CGT CAG TGA 3'and 5'CTG GCG CAT AGG GCA CTT 3'. The gene-specific primers of human housekeeping gene GAPDH were 5'AAC AGC CTC AAG ATC ATC AGC 3'and 5'GGA TGA TGT TCT GGA GAG CC 3'. Only those samples with a positive GAPDH amplification were used for this study.

Flow cytometry. All blood samples were processed on the day of collection and the antibodies used in this study were purchased from BD Biosciences and R\&D Systems. For the detection of natural GC B cells, PBMC were stained with peridinin chlorophyll protein (PerCP)-conjugated anti-CD19, fluorescein isothiocyanate (FITC)-conjugated anti- 
Table 2. Association of cell frequency with HBV-induced diseases

\begin{tabular}{|c|c|c|c|c|}
\hline \multirow[t]{2}{*}{ Trait } & \multicolumn{2}{|c|}{ CD19+CD3- } & \multicolumn{2}{|c|}{$\mathrm{CD}^{2}{ }^{+} \mathrm{CD}^{3} 8^{+} \mathrm{IgD}^{-}$} \\
\hline & Mean \pm SD (\%) & p & Mean \pm SD $(\%)$ & $\mathrm{p}$ \\
\hline $\mathrm{HC}(\mathrm{n}=27)$ & $6.2 \pm 2.5$ & - & $0.8 \pm 0.3$ & - \\
\hline $\mathrm{CHB}(\mathrm{n}=25)$ & $8.4 \pm 5.1$ & 0.042 & $1.1 \pm 0.5$ & 0.031 \\
\hline $\operatorname{LC}(\mathrm{n}=20)$ & $15.4 \pm 9.3$ & 0.017 & $3.7 \pm 1.6$ & 0.004 \\
\hline $\operatorname{HCC}(n=20)$ & $14.6 \pm 8.5$ & 0.023 & $4.2 \pm 2.8$ & 0.008 \\
\hline
\end{tabular}

$\mathrm{p}$ values compared the patients group to the healthy controls group

-CD38 and phycoerythrin (PE)-conjugated anti-IgD and gating was on CD19 B cells. For the detection of FcRY positively expressed B cells, PBMC was first fixed and permeabilized using 4\% paraformaldehyde and ice-cold methanol and then stained with PerCP-conjugated anti-CD19 and FITC-conjugated anti-FcRY. After staining, cells were washed twice and resuspended in fluorescence-activated cell sorting solution, fixed in PBS containing 1\% paraformaldehyde, and analyzed in FACSCalibur system. Mouse isotype-matched FITC, PE and PerCP were used as negative controls.

Statistical analysis. Demographic and clinical data were presented as mean \pm SD deviation. Differences between variables were compared by the unpaired Student's $t$-test, and differences in proportions were compared by Fisher's exact test as appropriate. A p-value of $<0.05$ was considered to be significant. Analysis of data was performed using SPSS 13.0 (Chicago, IL, USA).

\section{Results}

\section{Association of $B$ cell frequency with $\mathrm{HBV}$-induced diseases}

PBMC from healthy controls and HBV patients was analyzed using flow cytometry for evidence of chronic antigenic stimulation. First, the percentage of B cells from PBMC in the four studied groups was investigated by staining with anti-CD19 and anti-CD3. In controls, the frequency of CD19 B cells ranged from $3.22 \%$ to $15 \%$ of gated lymphocytes. The range in HBV patients was much greater, with CD19 B cell frequencies ranging between $2.52 \%$ and $30.1 \%$ of gated lymphocytes. The variance of the HBV patients' $B$ cell frequency data was significantly different from that for the healthy controls $(\mathrm{p}<0.05)$ but there was no significant difference among patients of different groups (Table 2).

To further investigate possible alteration of GC B cells in different HBV-induced disease status, PBMC were stained for anti-CD38 and anti-IgD of gated CD19 B cells. A small proportion of GC B cells $\left(\mathrm{CD} 38^{+} \mathrm{IgD}^{-}\right)$was found circulating in peripheral blood and the percentage of GC B cells was significantly increased in the patients compared to healthy controls $(\mathrm{p}<0.01)$. We next questioned whether the percentage of GC B cells would vary within patients of different disease status. We found that the proportion of GC B cells of CHB patients was significantly different compared to the other two groups $(\mathrm{p}<0.05)$, but there was no significant difference between LC and HCC patients (Table 2).

\section{Expression profile of FcRY in different $H B V$-induced diseases}

FcRY mRNA expression was detectable by quantitative real-time PCR in 24 of 27 (89\%) normal samples and 63 of $65(97 \%)$ patient samples. Relative expression of FcRY mRNA levels in B cells from patients with HBV-induced diseases and healthy controls was calculated by normalizing against GAPDH. Significantly higher levels were found in the HBV patients compared to healthy donors. Furthermore, we found FcRY expression in CHB patients was about 20 -fold higher $(\mathrm{p}<0.05)$. However, in patients with HBV-associated LC and HCC, FcRY was about 500-fold higher $(\mathrm{p}<0.01)$, compared to healthy controls. In addition, there was a significant difference for expression of FcRY gene between CHB patients and the other two groups $(p<0.05)$, but no significant difference between LC and HCC patients (Figure 1).

Flow cytometric analysis of intracytoplasmic expression of FcRY protein was performed in B cells from 87 clinical samples including 24 healthy controls and $63 \mathrm{HBV}$-infected patients (Figure 2). As expected, FcRY protein expression was observed in 87 samples. The FcRY levels on the CD19 B cells in HCC $(45.0 \% \pm 15.1 \%)$ and LC $(41.7 \% \pm 13.3 \%)$ patients were significantly higher compared to $\mathrm{CHB}$ patients $(11.4 \pm 4.1 \%, \mathrm{p}<0.05)$ and healthy controls $(2.5 \pm 1.2 \%, \mathrm{p}<0.01)$. Overall, the protein expression pattern was similar to that obtained by real-time PCR. 


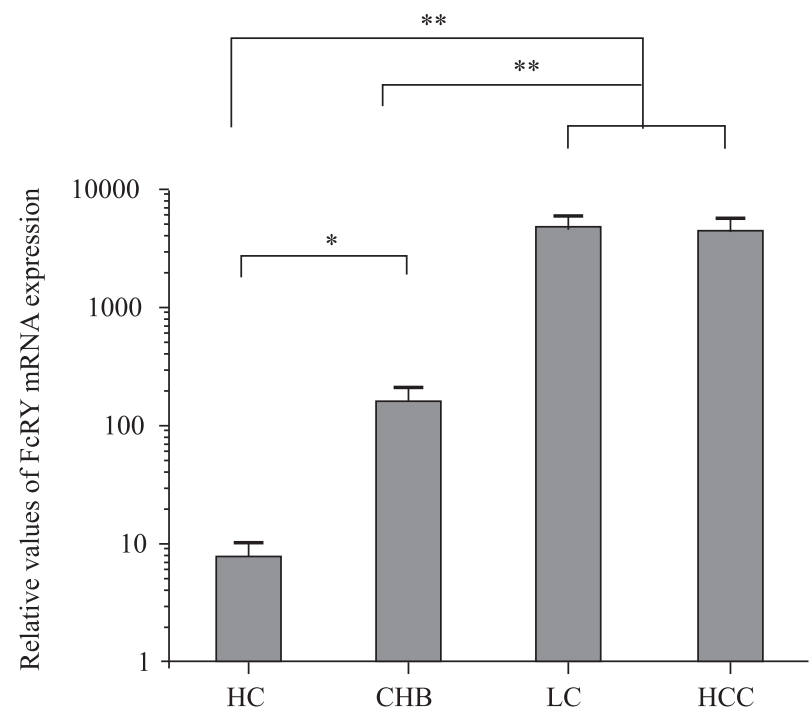

Figure 1. mRNA expression of FcRY on B lymphocytes from peripheral blood in patients with $\mathrm{HBV}$-induced diseases and healthy controls; ${ }^{*} \mathrm{p}<0.05 ;{ }^{*} \mathrm{p}<0.01$
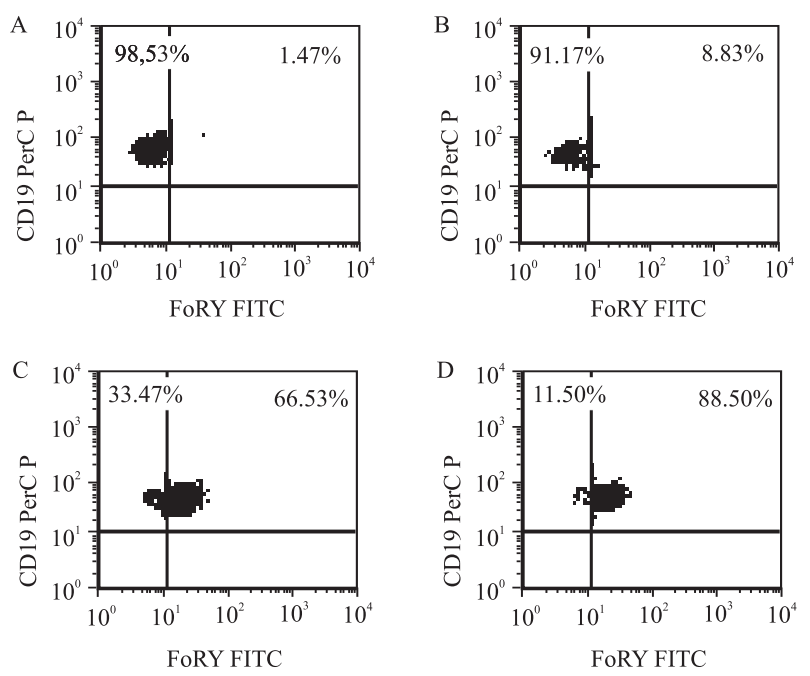

Figure 2. Protein expression of FcRY on B lymphocytes from peripheral blood in patients with $\mathrm{HBV}$-induced diseases and healthy controls. A: healthy control; B: CHB patient; C: LC patient; D: HCC patient

These results indicated that the protein expression level of FcRY was indeed correlated positively with their corresponding mRNA expression level (Figure 3). The expression level of FcRY was significantly associated with the progression of HBV-related diseases.

\section{Discussion}

$\mathrm{HBV}$ infection is a serious problem worldwide, especially in China. A substantial number of HBV carriers develop cirrhosis and HCC, but the mechanism underlying the different outcomes among patients is

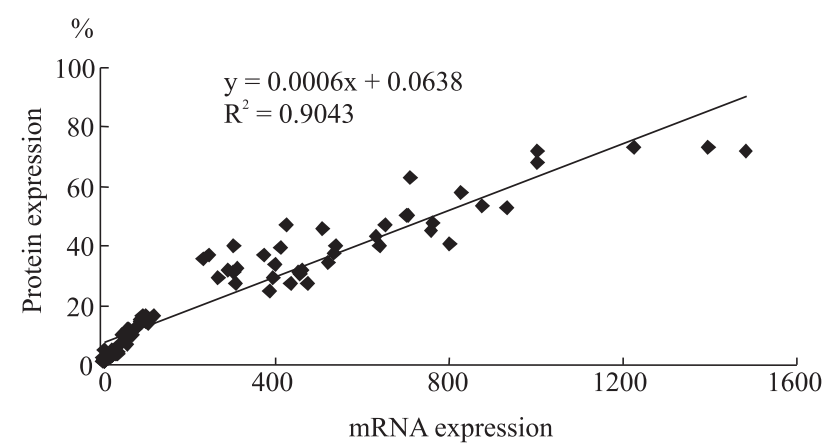

Figure 3. Comparision of mRNA and protein expression level of FcRY in 87 subjects. The real-time PCR results represent the relative values of FcRY mRNA expression to that of GAPDH multiplied by 100,000 . The flow cytometry results are presented as percentage

not clear [1-3]. In this study, we investigated the expression profile of FcRY and B lymphocytes population in HBV-induced diseases to help clarify one aspect of the function of FcRY, which was over-expressed in HBV-induced diseases. Previous studies failed to reveal any FcRY mRNA expression in patients with autoimmune diseases. However, expression of FcRY was found to be strongly enhanced in malignant and metastatic melanomas compared to that in melanocytic nevi [20]. To the best of our knowledge, this study is the first to report the correlation of FcRY expression profile, B lymphocytes population from peripheral blood, and different stages of HBV infection.

Since FcRY is preferentially expressed by B lymphocytes and largely limited to the germinal center B cells, we measured the frequency of B lymphocytes and germinal center B cell subpopulation of HBV-infected patients compared to healthy controls. The percentage of the HBV patients' B cells in PBMC was significantly higher than that of the healthy controls. Furthermore, the germinal center B cell subpopulation was also increased in the course of HBV infection. The elevated presentation of this subpopulation might be the result of $\mathrm{B}$ cell activation in this disease. Then we measured the mRNA and protein expression level of FcRY in 92 samples including HBV-related patients and healthy donors.

The results showed FcRY expression increased as the HBV-induced disease progressed from chronic hepatitis to cirrhosis and HCC. In addition, up-regulation of FcRY was indeed correlated positively with their corresponding mRNA expression level. The rarity of B cells expressing the FcRY gene in the blood of healthy donors may be explained by the low percentage of GC B cells in the peripheral blood of healthy donors and an increased expression level of FcRY may correlate with change of GC B cells in 
peripheral blood under the pathological progression. However, the mechanism by which HBV antigen affects the function of B lymphocytes is unclear and needs to be addressed in future studies.

In conclusion, the results of our study suggest that B lymphocytes are more active in HBV-induced diseases because of the increased proportion of GC $B$ cells. And the detection of increased FcRY mainly expressed on GC B cells is associated with a high risk of HBV chronic infection development into cirrhosis and HCC. So FcRY is perhaps one of the B cell activation markers.

However, it is not yet clear if activation of B lymphocytes and up-expression of FcRY are the cause, or the outcome, of the disease progression. Additional studies are underway to elucidate the molecular mechanism and the more detailed function of FcRY and B lymphocytes in $\mathrm{HBV}$-induced diseases.

\section{Acknowledgements}

The authors are very grateful to the Natural Science Foundation of Jiangsu Province (BK2008530).

\section{References}

1. Lavanchy D. Hepatitis B virus epidemiology, disease burden, treatment, and current and emerging prevention and control measures. $J$ Viral Hepat. 2004;11:97-107.

2. Gish RG. Hepatitis B treatment: current best practices, avoiding resistance. Cleve Clin J Med. 2009;76:S14-S19.

3. Bertoletti A, Gehring AJ. The immune response during hepatitis B virus infection. $J$ Gen Virol. 2006;87:1439-1449.

4. Rehermann B, Nascimbeni M. Immunology of hepatitis B virus and hepatitis $\mathrm{C}$ virus infection. Nat Rev Immunol. 2005;5:215-229.

5. Jung MC, Diepolder HM, Pape GR. T cell recognition of hepatitis B and C viral antigens. Eur J Clin Invest. 1994;24: 641-650.

6. Wang NS, Wu ZL, Zhang YE, Guo MY, Liao LT. Role of hepatitis B virus infection in pathogenesis of IgA nephropathy. World J Gastroenterol. 2003;9:2004-2008.
7. Davis RS, Wang YH, Kubagawa H, Cooper MD. Identification of a family of $\mathrm{Fc}$ receptor homologs with preferential B cell expression. Proc Natl Acad Sci USA. 2001;98:9772-9777.

8. Davis RS, Dennis G Jr, Odom MR et al. Fc receptor homologs: newest members of a remarkably diverse Fc receptor gene family. Immunol Rev. 2002;190:123-136.

9. Mechetina LV, Najakshin AM, Volkova OY et al. FCRL, a novel member of the leukocyte Fc receptor family possesses unique structural features. Eur J Immunol. 2002;32:87-96.

10. Ehrhardt GR, Leu CM, Zhang S et al. Fc receptor-like proteins (FCRL): immunomodulators of B cell function. $A d v$ Exp Med Biol. 2007;596:155-162.

11. Leu CM, Davis RS, Gartland LA, Fine WD, Cooper MD. FcRH1: an activation coreceptor on human B cells. Blood. 2005;105:1121-1126.

12. Li FJ, Ding S, Pan J et al. FCRL2 expression predicts IGHV mutation status and clinical progression in chronic lymphocytic leukemia. Blood. 2008;112:179-187.

13. Nagata S, Ise T, Pastan I. Fc receptor-like 3 protein expressed on IL-2 nonresponsive subset of human regulatory T cells. J Immunol. 2009;182:7518-7526.

14. Ehrhardt GRA, Hijikata A, Kitamura H, Ohara O, Wang JY, Cooper MD. Discriminating gene expression profiles of memory B cell subpopulations. J Exp Med. 2008;205:1807-1817.

15. Haga CL, Ehrhardt GRA, Boohaker RJ, Davis RS, Cooper MD. Fc receptor-like 5 inhibits B cell activation via SHP-1 tyrosine phosphatase recruitment. Proc Natl Acad Sci USA. 2007;104:9770-9775.

16. Mohan J, Dement-Brown J, Maier S, Ise T, Kempkes B, Tolnay M. Epstein-Barr virus nuclear antigen 2 induces FcRH5 expression through CBF1. Blood. 2006;107:4433-4439.

17. Davis RS, Li H, Chen CC, Wang YH, Cooper MD, Burrows PD. Definition of an Fc receptor-related gene (FcRX) expressed in human and mouse B cells. Int Immunol. 2002; 14:1075-1083.

18. Masuda K, Davis RS, Maruyama T et al. FcRY, an Fc receptor related gene differentially expressed during $\mathrm{B}$ lymphocyte development and activation. Gene. 2005;363:32-40.

19. Wilson TJ, Colonna M. A new Fc receptor homolog, FREB2, found in germinal center B cells. Genes Immun. 2005;6:341-346.

20. Chikaev NA, Bykova EA, Najakshin AM et al. Cloning and characterization of the human FCRL2 gene. Genomics. 2005;85:264-272.

21. Bhogal RK, Bona CA. B cells: no longer bystanders. J Clin Invest. 2005;115:2962-2965.

Submitted: 15 November, 2011 Accepted after reviews: 15 February, 2011 